\title{
Functional outcomes in ICU - what should we be using? - an observational study
}

Selina M Parry ${ }^{1 *}$, Linda Denehy ${ }^{1,3}$, Lisa J Beach², Sue Berney ${ }^{3,4}$, Hannah C Williamson ${ }^{4}$ and Catherine L Granger ${ }^{1,2,3}$

\begin{abstract}
Introduction: With growing awareness of the importance of rehabilitation, new measures are being developed specifically for use in the intensive care unit (ICU). There are currently 26 measures reported to assess function in ICU survivors. The Physical Function in Intensive care Test scored (PFIT-s) has established clinimetric properties. It is unknown how other functional measures perform in comparison to the PFIT-s or which functional measure may be the most clinically applicable for use within the ICU. The aims of this study were to determine (1) the criterion validity of the Functional Status Score for the ICU (FSS-ICU), ICU Mobility Scale (IMS) and Short Physical Performance Battery (SPPB) against the PFIT-s; (2) the construct validity of these tests against muscle strength; (3) predictive utility of these tests to predict discharge to home; and (4) the clinical applicability. This was a nested study within an ongoing controlled study and an observational study.
\end{abstract}

Methods: Sixty-six individuals were assessed at awakening and ICU discharge. Measures included: PFIT-S, FSS-ICU, IMS and SPPB. Bivariate relationships (Spearman's rank correlation coefficient) and predictive validity (logistic regression) were determined. Responsiveness (effect sizes); floor and ceiling effects; and minimal important differences were calculated.

Results: Mean \pm SD PFIT-s at awakening was $4.7 \pm 2.3$ out of 10 . On awakening a large positive relationship existed between PFIT-s and the other functional measures: FSS-ICU (rho $=0.87, p<0.005$ ), IMS (rho $=0.81, p<0.005$ ) and SPPB ( $r h o=0.70, p<0.005)$. The PFIT-s had excellent construct validity (rho $=0.8, p<0.005)$ and FSS-ICU (rho $=0.69, p<0.005)$ and IMS ( $r$ ho $=0.57, \mathrm{p}<0.005$ ) had moderate construct validity with muscle strength. The PFIT-s and FSS-ICU had small floor/ceiling effects $<11 \%$ at awakening and ICU discharge. The SPPB had a large floor effect at awakening (78\%) and ICU discharge (56\%). All tests demonstrated responsiveness; however highest effect size was seen in the PFIT-s (Cohen's $d=0.71)$.

Conclusions: There is high criterion validity for other functional measures against the PFIT-s. The PFIT-s and FSS-ICU are promising functional measures and are recommended to measure function within the ICU.

Trial registration: Clinicaltrials.gov NCT02214823. Registered 7 August 2014).

\section{Introduction}

Impairment in physical function is a significant problem for survivors of critical illness [1-3]. Physical function refers to 'the ability to carry out various activities that require physical capability ranging from self-care to more vigorous activities that require increasing degrees of mobility, strength or endurance' [4]. The International Classification of Functioning (ICF) framework provides a conceptual model to guide patient assessment, which

\footnotetext{
* Correspondence: parrys@unimelb.edu.au

${ }^{1}$ Department of Physiotherapy, The University of Melbourne, Level 7 Alan Gilbert Building, 161 Barry St, Parkville, 3010 Melbourne, VIC, Australia Full list of author information is available at the end of the article
}

includes examination of impairment, activity limitations and participation restrictions [2,5]. In survivors of critical illness, measurement of physical function using performance-based tests provides information on the patients' activity limitations. There are currently 26 selfreport and performance-based measures reported in the literature to assess physical function in ICU survivors [6].

When selecting the most appropriate outcome measure to assess physical function, clinicians and researchers need to consider which outcome measures have robust clinimetric properties [2]. This includes the ability of an outcome measure to measure what it is intended to measure, that is, how well the test results relate to data 
obtained from the gold standard instrument (criterionconcurrent validity); how well the outcome obtains data, as hypothesized, when compared to an instrument measuring a similar construct (convergent or construct validity); or how well data predict an outcome (predictive validity/utility) $[7,8]$. Additionally, the clinical applicability of the outcome measure is also important. This includes whether there is a floor or ceiling effect; the ability of the outcome measure to detect meaningful change over time (responsiveness) [8]; and whether there is a known minimal important difference (the smallest change in the outcome measure that patients and clinicians consider to be clinically relevant) [9]. These clinimetric properties should be examined specifically within the setting in which the outcome measure will be used [10]. This is particularly important for a challenging environment such as ICU, where fluctuations in patient mental alertness, ability to follow commands, and both rapid changes in medical stability and a confined space may impact on the choice, reliability and validity of outcome measures $[2,11,12]$.

Whilst 26 different functional measures have been described for use within critically ill patients, there are currently only six published functional measures that have been developed specifically for the ICU setting and have undergone clinimetric evaluation [6]. These measures are the Physical Function in Intensive care Test scored (PFIT-s) [13], Chelsea Critical Care Physical Assessment tool (CPAx) [14], Perme mobility scale [15], Surgical intensive care unit Optimal Mobilization Score (SOMS) [16], ICU Mobility Scale (IMS) [17], and the Functional Status Score for the ICU (FSS-ICU) [18,19]. To date, studies of clinimetric properties have been primarily limited to a single component, such as reliability testing [6]. There is also growing interest in the application of the Short Physical Performance Battery (SPPB) [20] or its components [21] to evaluate functional recovery in individuals with critical illness. The SPPB is a battery tool, originally developed for use in the geriatric population $[22,23]$ and may discriminate between individuals with critical illness who receive early rehabilitation versus usual care [20]. However, there have been no published studies on the SPPB examining specifically the clinimetric properties of this test within the ICU setting.

The clinimetric properties of functional measures for use within the ICU setting are still in early development. The PFIT-s [13,24] and CPAx tools [14,25] have the most established clinimetric properties in terms of reliability, validity and responsiveness. The PFIT-s can be used to guide exercise prescription within the ICU as well as measuring functional recovery $[13,26]$. The PFIT-s can be used as a reference standard against which other functional measures can be compared.

Three functional measures, which were selected for comparison against the PFIT-s within this study, were:
FSS-ICU, IMS and the SPPB. It is unknown how these other functional measures perform in comparison to the PFIT-s or which of these four functional measures may be the most clinically applicable for use within the ICU. Therefore, the aims of this study were to determine (1) the criterion validity of the FSS-ICU, IMS and SPPB against the PFIT-s; (2) the construct validity of these outcome measures against measurement of muscle strength; (3) the predictive utility of these outcome measures to predict patients who would discharge directly home from hospital; and (4) the clinical applicability of the outcome measures (floor and ceiling effects, responsiveness and minimal important difference). It was hypothesized that the FSS-ICU would have the strongest positive correlation with the PFIT-s (correlation $>0.50$ ). The COnsensus-based Standards for the selection of health status Measurement INstruments (COSMIN) guidelines [27] and the Strengthening the Reporting of Observational Studies in Epidemiology (STROBE) guidelines [28] were followed in reporting this study.

\section{Materials and methods \\ Study design, setting and participants}

This was a nested observational study within an ongoing controlled trial (NCT02214823) and an observational study conducted in major mixed (medical and surgical) ICUs in Melbourne, Australia. Consecutive participants were recruited between July 2012 and June 2014 for the two main trials. Both sites had institutional ethical approval (Melbourne Health and Austin Health Human Research Ethics Committees) and participants provided written informed consent. Participants were included in the primary trials if they were admitted to an ICU, were more than 18 years old, English speaking, and were mechanically ventilated for 48 hours and expected to remain in the unit for at least four days. Patients were excluded from the primary trials if they had a premorbid physical or cognitive impairment that would prevent exercise, or were admitted with a new neurological insult such as stroke or spinal cord injury. All patients were required to be able to ambulate at least 10-metres independently prior to ICU admission (+/- gait aid). Patients were included in the nested study as a convenience sample if they had been assessed at both awakening and ICU discharge.

\section{Procedure}

Assessments were performed twice in the ICU for each patient: on awakening and ICU discharge. Day of awakening was defined as when the patient scored greater than three out of five on the De Jonghe comprehension criteria on two consecutive occasions within a 6-hour period [29]. Between the two time points of testing (awakening and ICU discharge), patients received usual- 
care rehabilitation involving early mobility activities such as active exercises in bed, sitting on the edge of the bed, standing, marching, and walking. At each time point of testing, the participants completed the PFIT-s and a range of additional functional tests (FSS-ICU, IMS, SPPB). The Medical Research Council-Sum Score (MRC-SS) [30] was assessed at awakening in order to determine the incidence of ICU-acquired weakness (ICU-AW) within the population studied. The order of testing varied, and was not controlled within this study. In order to minimize fatigue as an issue, all tests were completed within a 12-hour period, and participants were required to return to baseline based on clinical parameters such as heart rate, respiratory rate and oxygen saturation levels before the next functional test was undertaken. Patients were stable and unchanged in the time between completing the PFIT-s and additional functional tests. All assessors were qualified physical therapists and had received standardized training in the outcome measures (PFIT-s, SPPB, FSS-ICU, IMS, MRC-SS) from one senior physical therapist. The same assessor (where possible) performed awakening and ICU discharge measures within the same patient.

\section{Physical function in intensive care test scored}

The PFIT-s is a battery outcome measure involving four components: sit to stand assistance, marching on the spot cadence, shoulder flexor and knee extensor strength. The PFIT-s scores range from 0 (able to perform strength testing only with a maximum score of 2 out of 5 for shoulder and knee) to 10 (performance without any difficulty) $[13,31]$. This tool has established validity, reliability and a minimal clinically important difference (MCID) of 1.5 points out of 10 [13,31].

\section{Additional outcome measures}

Participants also completed three additional functional measures: the FSS-ICU, IMS and SPPB. The FSS-ICU involves assessment of five functional tasks (rolling, supine to sit transfers, unsupported sitting, sit to stand transfers, and ambulation). The five tasks are scored on a seven-point scoring system from the Functional Independence Measure $[18,19]$. Higher scores represent better function and the total score ranges from 0 to 35 . The FSS-ICU was originally developed for use within the ICU setting [18] however there has been no evaluation of the clinimetric properties of the FSS-ICU specifically within the ICU setting. Despite this the FSS-ICU has been shown to be responsive to change over time and a valid predictor of discharge destination when implemented in a long-term acute care facility [19].

The IMS is an 11-item categorical scale that rates the patient's highest level of mobility, where $0=$ nothing and $10=$ walking independently without a gait aid [17]. Although there is high inter-rater reliability for this measure in the ICU setting, there are no published data on other clinimetric properties (validity, responsiveness) [17].

The SPPB is a battery tool, which was originally developed for use in the geriatric population [22,23]. A score of 0 to 12 (higher scores indicating better function) is based on the performance of three tasks: gait speed, chair rise time (five times sit to stand) and standing balance (tandem, semi-tandem and side by side) [22]. This is increasingly being used in the ICU setting and to date there are no published data on the clinimetric properties or clinical applicability of the SPPB within the ICU setting.

Baseline demographics were recorded, including age, sex, body mass index, admission diagnosis and severity of illness (Acute physiological and chronic health evaluation (APACHE) II within first 24 hours of ICU admission). Additionally ICU length of stay (LOS), mechanical ventilation (MV) duration in days, and acute hospital discharge destination were recorded.

\section{Sample size}

The sample size was 66 . Sample sizes of $\geq 50$ participants are recommended for studies assessing clinimetric properties of measurements to enhance the generalizability of findings [32,33]. The examination of the SPPB was performed in a subgroup $(\mathrm{n}=23)$ within this nested study. Only participants from one of the two trials completed the SPPB. Therefore SPPB analyses are underpowered and results should be viewed with caution. The measurement of SPPB was opportunistic to enable preliminary examination of the clinimetric properties of the SPPB in individuals with critical illness, which had not been reported within the literature to date.

\section{Statistical analyses}

Data were analysed with SPSS Windows Version 22.0 (SPSS, Chicago, IL, USA). Data were assessed for normality using the Kolmogorov-Smirnov statistic. Parametric data are presented as mean and SD, and non-parametric data are presented as median and IQR. Spearman's rank correlation coefficient was used to assess the bivariate relationships between test scores (PFIT-s, FSS-ICU, IMS and SPPB) [7]. Coefficients were interpreted as: little (0.00 to 0.25 ), fair ( 0.25 to 0.50$)$, moderate ( 0.50 to 0.75 ) and excellent association (0.75 to 1.0) [8]. Alpha was set at 0.05 for all analyses.

Predictive utility of the tests were assessed using logistic regression analyses to investigate the ability of the test to predict likelihood of discharge directly to home compared to other destinations. Logistic regression analyses were run separately for the PFIT-s, FSS-ICU and IMS on awakening and ICU discharge (SPPB was not assessed with logistic regression as this had no bivariate correlation with discharge destination). The test score 
on awakening was the variable of interest (independent variable) and was included in all regression models. The outcome of interest (dependent variable) was the dichotomous variable of discharge directly to home and was coded as yes (discharged directly home) or no (not discharged directly home). Potential covariates were: age (in years), sex (coded as male or female), body mass index (in $\mathrm{kg} / \mathrm{m}^{2}$ ), APACHE II (in points), MV duration (in days), MRC-SS on awakening (in points), and ICU LOS (in days). The potential covariates with significant bivariate correlation with the dependent variable were included in the model if collinearity was not identified. Collinearity was assessed using Spearman's rank correlation coefficient and defined as rho $\geq 0.7$. Overfitting of the model was avoided with no more than three independent variables included in the final model. Conformity to a linear gradient for variables was examined with inspection of a plot with locally weighted scatterplot smoothing (LOWESS). Goodness of fit was examined using the Hosmer-Lemeshow goodness of fit test and poor fit was defined as alpha $<0.05$. In addition the differences in test scores on awakening of those participants discharged directly home versus those discharged to other destinations were determined using the independent $t$-test for continuous parametric data, the Mann-Whitney $U$-test for ordinal non-parametric data and the Chi-square test for categorical data.

Floor and ceiling effects of the PFIT-s, FSS-ICU, IMS and SPPB were determined using the percentage of occasions when participants scored the lowest or highest score possible for the test. Change over time, from awakening to ICU discharge, was assessed using the paired $t$-test for parametric data [8] and the Wilcoxon signed rank test for non-parametric data [8]. Responsiveness of each test was determined with calculation of the effect size. For parametric data this was defined as Cohen's $d$ and calculated as the mean difference divided by pooled SD [34]. For non-parametric data this was defined as $r=Z$ divided by the square root of the sample size [8,34]. Thresholds for interpretation of the change were: small (0.2 to 0.49$)$, moderate $(0.5$ to 0.79$)$ and large $(\geq 0.8)[34,35]$.

The minimal important differences (MIDs) of the continuous and ordinal tests were determined using distribution-based estimation with calculation of the standard error of the measurement (SEM) and effect size (ES). The SEM was calculated as $\sigma_{1} \sqrt{ }(1-r)$, where $\sigma_{1}$ was the baseline $\mathrm{SD}$ of the test score and $r$ was the test-retest reliability coefficient of the test [36]. A moderate ES is considered a clinically important effect and was calculated using the formula $0.5 \times \mathrm{SD}$ of the change scores [37].

\section{Results}

The PFIT-s and additional functional tests were conducted with 66 patients. The characteristics of the cohort studied are reported in Table 1. All participants were previously independent and prior to hospitalization in the ICU were from home. The mean \pm SD PFIT-s on awakening was $4.7 \pm 2.3$ out of 10 (Table 1 ).

\section{Validity}

There was moderate to large criterion validity between the PFIT-s and the three other functional tests (Figure 1). On awakening large positive relationships existed between PFIT-s and the FSS-ICU $(n=66$, rho $=0.87,95 \%$

Table 1 Demographics of the cohort

\begin{tabular}{|c|c|}
\hline Variable & $\begin{array}{l}\text { Total sample } \\
(\mathrm{n}=66)\end{array}$ \\
\hline Male, n (\%) & $40(61 \%)$ \\
\hline Age, mean $\pm S D$ & $58 \pm 17$ \\
\hline Body mass index, $\mathrm{kg} / \mathrm{m}^{2}$ median (IQR) & 28 (24 to 32 ) \\
\hline $\begin{array}{l}\text { Acute physiological and chronic health evaluation } \|_{\text {, }} \\
\text { score }\end{array}$ & $21 \pm 7$ \\
\hline \multicolumn{2}{|l|}{ ICU admission diagnosis } \\
\hline - respiratory, n (\%) & $14(21 \%)$ \\
\hline - gastrointestinal, n (\%) & $12(18 \%)$ \\
\hline - sepsis, non-pulmonary, n (\%) & $13(20 \%)$ \\
\hline - cardiac, n (\%) & $18(27 \%)$ \\
\hline - trauma, n (\%) & $5(8 \%)$ \\
\hline - other, n (\%) & $4(6 \%)$ \\
\hline $\begin{array}{l}\text { Medical Research Council (MRC) sum-score on } \\
\text { awakening median (IQR) }\end{array}$ & 48 (39 to 54$)$ \\
\hline ICU-acquired weakness (<48/60 MRC) on awakening & $28(42 \%)$ \\
\hline ICU length of stay, days, median (IQR) & $8(5$ to 15$)$ \\
\hline Mechanical ventilation time, days, median(IQR) & $5(3$ to 10$)$ \\
\hline \multicolumn{2}{|l|}{ Acute hospital discharge destination } \\
\hline - home, n (\%) & $37(56 \%)$ \\
\hline - inpatient rehabilitation facility, n (\%) & $20(30 \%)$ \\
\hline - deceased in hospital, n (\%) & $3(4 \%)$ \\
\hline - other or unknown, n (\%) & $6(9 \%)$ \\
\hline $\begin{array}{l}\text { Physical function in ICU test scored, on awakening, } \\
\text { mean } \pm \text { SD }\end{array}$ & $4.7 \pm 2.3$ \\
\hline $\begin{array}{l}\text { Physical function in ICU test scored, at ICU discharge, } \\
\text { mean } \pm S D\end{array}$ & $6.3 \pm 2.2$ \\
\hline
\end{tabular}

Functional status score for ICU, on awakening,

$12.0(6.0$ to 17.0$)$ median (IQR)

Functional status score for ICU, at ICU discharge, median (IQR)

$17.0(12.0$ to 26.5$)$

ICU mobility scale, on awakening, median (IQR)

$3.5(1.0$ to 5.0$)$

ICU mobility scale, at ICU discharge, median (IQR)

6.0 (5.0 to 8.0$)$

Short Physical Performance Battery, on awakening, median (IQR) $(n=23)$

$0.0(0.0$ to 0.0$)$

Short Physical Performance Battery, at ICU discharge, $\quad 0.0$ (0.0 to 5.0) median (IQR) $(n=23)$

${ }^{\mathrm{a} A P A C H E}$ II score was determined within the first 24 hours of ICU admission. 

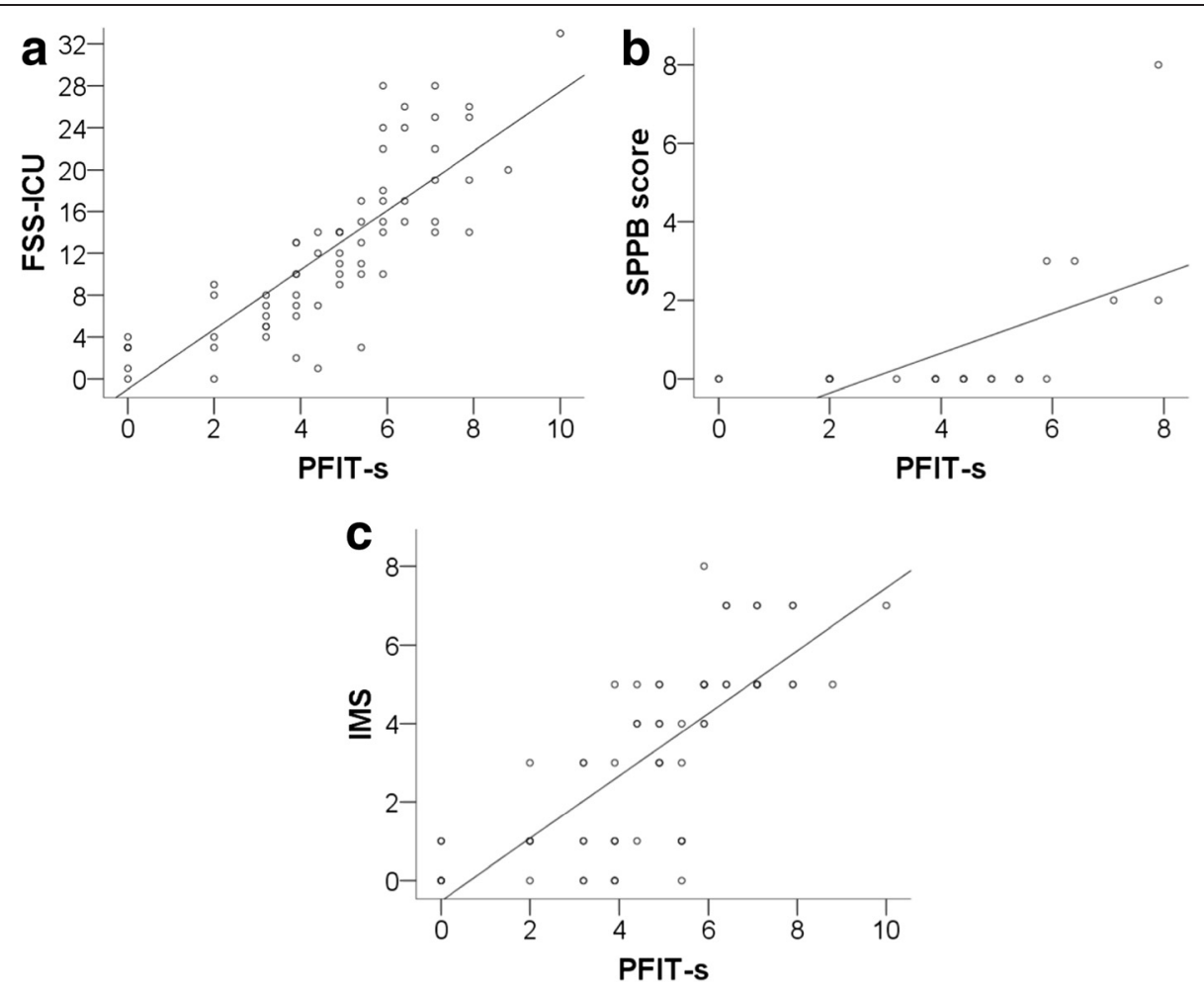

Figure 1 Relationship between physical function in intensive care test scored (PFIT-s) and (a) Functional Status Score for the Intensive Care Unit (FSS-ICU), (b) Short Physical Performance Battery (SPPB) and (c) ICU mobility scale (IMS) on awakening.

$\mathrm{CI}=0.79,0.92, P<0.005)$ (Figure 1a) and IMS $(\mathrm{n}=66$, rho $=0.81,95 \% \mathrm{CI}=0.70,0.88, \mathrm{P}<0.005$ ) (Figure 1c); and a moderate positive relationship existed between the PFIT-S and SPPB $(\mathrm{n}=23$, rho $=0.70,95 \% \mathrm{CI}=0.47,0.83$, $P<0.005)$ (Figure 1b). At ICU discharge large positive relationships existed between PFIT-s and FSS-ICU $(n=66$, rho $=0.85,95 \% \mathrm{CI}=0.77,0.90, P<0.005)$ and SPPB $(\mathrm{n}=23$, rho $=0.86,95 \% \mathrm{CI}=0.73,0.91, P<0.005)$; and a moderate positive relationship existed between the PFIT-s and IMS (n $=64$, rho $=0.66,95 \% \mathrm{CI}=0.49,0.80, P<0.005)$.

The PFIT-s demonstrated excellent construct validity with measurement of muscle strength: on awakening a large positive relationship existed between the PFIT-s and MRC sum-score $(\mathrm{n}=66$, rho $=0.8,95 \% \mathrm{CI}=0.68$, $0.89, P<0.005)$. In addition, moderate positive relationships existed between FSS-ICU and MRC sum-score $(\mathrm{n}=66$, rho $=0.69,95 \% \mathrm{CI}=0.50,0.83, P<0.005)$, and between IMS and MRC sum-score $(\mathrm{n}=66$, rho $=0.57,95 \% \mathrm{CI}=$ $0.36,0.74, P<0.005)$. There was no relationship between SPPB and MRC sum-score on awakening $(\mathrm{n}=23$, rho $=$ $0.30,95 \% \mathrm{CI}=-0.08,0.62, P=0.161)$.

\section{Predictive utility}

Fifty-six percent $(n=37)$ of participants were discharged directly home from their acute hospital stay (Table 1). Higher PFIT-s scores on awakening (odds ratio $(\mathrm{OR})=1.59, P=0.004)$ and lower age were significant factors in determining whether the patient was discharged directly home (Additional file 1: Table S1); this was not found for FSS-ICU $(P=0.642)$ or IMS $(P=$ 0.143 ) when assessed on awakening. Lower age and higher test scores at ICU discharge were significant factors in determining discharge to home: PFIT-s (OR $=1.56, P=$ 0.005), FSS-ICU $(\mathrm{OR}=1.09, P=0.013)$ and IMS (OR $=$ $1.54, P=0.011$ ) (Additional file 1: Table S1). There was a significant difference in the PFIT-s, IMS and FSS-ICU scores on awakening between participants who were discharged home from the acute hospital versus those who were discharged to another location $(P<0.05)$, this was not the case for the SPPB $(P>0.05)$.

\section{Clinical applicability}

Table 2 provides the floor and ceiling effects for each test, as well as the range of test scores at both awakening and ICU discharge.

There were significant improvements from awakening to ICU discharge in the PFIT-s (mean difference $=1.59,95 \%$ $\mathrm{CI}=1.12,2.06, P<0.005)$, FSS-ICU $(Z=-5.34, P<0.005)$, IMS $(Z=-6.71, \mathrm{P}<0$ 0.005) and SPPB $(Z=-2.23, \mathrm{p}=$ 0.026). All tests demonstrated responsiveness to change, however the highest effect size was seen for the PFIT-s: The effect sizes of the PFIT-s and IMS were 0.71 and 0.59 
Table 2 Floor and ceiling effects

\begin{tabular}{llll}
\hline Test & $\begin{array}{l}\text { Floor effect } \mathbf{n} / \text { total } \\
\mathbf{n} \text { assessed (\%) }\end{array}$ & $\begin{array}{l}\text { Ceiling effect } \mathbf{n} / \text { total } \\
\mathbf{n} \text { assessed (\%) }\end{array}$ & $\begin{array}{l}\text { Range of test scores } \\
\text { at each time point }\end{array}$ \\
\hline PFIT-S on awakening & $6 / 66(9.1 \%)$ & $1 / 66(1.5 \%)$ & 0 to 10 out of 10 \\
PFIT-s at ICU discharge & $1 / 66(1.5 \%)$ & $7 / 66(10.6 \%)$ & 0 to 10 out of 10 \\
FSS-ICU on awakening & $2 / 66(3.0 \%)$ & $0 / 66(0 \%)$ & 0 to 33 out of 35 \\
FSS-ICU at ICU discharge & $0 / 66(0 \%)$ & $2 / 66(3.0 \%)$ & 2 to 35 out of 35 \\
IMS on awakening & $11 / 66(16.7 \%)$ & $0 / 66(0 \%)$ & 0 to 8 out of 10 \\
IMS at ICU discharge & $0 / 64(0 \%)$ & $3 / 64(4.7 \%)$ & 1 to 10 out of 10 \\
SPPB on awakening & $18 / 23(82.6 \%)$ & $0 / 23(0 \%)$ & 0 to 8 out of 12 \\
SPPB at ICU discharge & $13 / 23(56.5 \%)$ & $0 / 23(0 \%)$ & 0 to 11 out of 12 \\
\hline
\end{tabular}

FSS-ICU, Functional Status Score for the Intensive Care Unit; IMS, ICU mobility scale; n, number; PFIT-s, Physical Function in Intensive Care Test scored; SPPB, Short Physical Performance Battery.

respectively, which represent a moderate responsiveness to change. The effect sizes of the FSS-ICU and SPPB were 0.46 and 0.33 respectively, which represent small responsiveness to change.

The minimal important differences for the three tests were estimated to be between 1.0 to 1.4 points for the PFIT-s, 4.3 to 5.6 points for the FSS-ICU, and 1.5 to 1.3 points for the SPPB according to calculation of the ES and SEM respectively.

\section{Discussion}

Physical function is an important outcome to measure in survivors of critical illness. In a moderately unwell cohort of ICU survivors from two mixed medical/surgical ICUs we found that there was high criterion validity for other functional measures against the PFIT-s especially for the FSS-ICU tool. The PFIT-s had strong construct validity with measurement of muscle strength, and both the FSS-ICU and IMS had moderate construct validity. There was no relationship between SPPB and muscle strength at awakening or ICU discharge. All functional measures except the SPPB were able to discriminate and predict future discharge destination.

The PFIT-s had high construct validity with muscle strength and was predictive of discharge destination when measured on awakening or at ICU discharge. This is in consensus with what has previously been reported within the ICU literature [13,31]. The MID for the PFIT-s was estimated to be between 1.0 to 1.4 points in this study, which was similar to that reported by Denehy and colleagues in 2013 (1.5 points out of 10.0) [13] adding further validity to the cutoff point previously developed. The floor and ceiling effects for the PFIT-s were small in our study ( 9 and $11 \%$ respectively). This is in contrast to previous studies, which reported a floor effect of 22 to $32 \%$ on awakening and a ceiling effect of 5 to $22 \%$ respectively $[13,31]$. The differences may in part be due to differences in patient cohort demographics and sedation practices and provisions of therapy within the units. In comparison to the study by Nordon-Craft and colleagues [31] there was a marked difference in overall MV duration and ICU LOS compared to our study (median MV duration of 12 days and ICU LOS of 20 days versus MV duration of 5 days and ICU LOS of 8 days). There was a large floor effect observed at baseline by Nordon-Craft and colleagues (32\%) [31] compared to our study with a floor effect of $9 \%$ on awakening. Similar to the findings of Denehy and colleagues [13] this paper identified that a higher PFIT-s (better function) predicted a greater likelihood of return to home. The demographics within the Australian study [13] were more comparable to the sample examined within our study.

Compared to the PFIT-s the FSS-ICU was the most robust functional outcome measure in the ICU setting. It was predictive of discharge destination when tested at ICU discharge and had small floor/ceiling effects within the ICU setting. Floor and ceiling effects are of concern for longitudinal studies as they limit the ability to detect change over time in terms of improvement and/or deterioration in functional recovery [7]. The floor effect observed at awakening was less than 15\% (the acceptable cutoff for outcome measures) [38] for the PFIT-s and FSS-ICU $(<10 \%)$. In contrast there was a large floor effect for SPPB on awakening and ICU discharge time points and a moderate floor effect at ICU awakening for IMS (17\%).

The SPPB is a high-level physical function outcome as it examines gait speed, balance control and sit-to-stand repetitions. Although power was not achieved for the SPPB outcome, large floor effects were observed for the SPPB suggesting it may not be a feasible measure for use within the ICU setting. Floor and ceiling effects are influenced by patient characteristics and therefore differences in sample characteristics may affect the choice of test used to evaluate physical function. For example the SPPB contains tasks requiring higher-level functional 
performance compared to the PFIT-s and FSS-ICU, which may be more sensitive in individuals with greater severity of illness and impairment to be able to detect change over time in the early ICU admission period. It is important that patient characteristics are considered when selecting a test. The SPPB may be more appropriate as a measure in the post ICU setting in the acute hospital wards or post hospital discharge.

The minimal important difference is the smallest change in an outcome that is considered to be clinically relevant [9]. There are two main methods used to determine the minimal important difference, the distributionbased method and the anchor-based method [39]. The distribution-based method utilizes statistical analyses to determine the minimal important difference using the degree of test score variability. The main disadvantage with this method is that it does not take into account whether the clinician or patient feels that change is clinically meaningful [40]. The anchor-based estimation takes into account a patient-related anchor such as the global rating of change scale to determine if the patient is clinically changed [39]. Future studies need to determine whether the cutoff values developed using the distribution-based methods remain stable when applying the anchor-based methods and demonstrate a change that is clinically meaningful.

Higher PFIT-s scores (better function) were shown to be predictive of discharge to home on both awakening and ICU discharge time points. Higher FSS-ICU and IMS scores were predictive of discharge to home only at ICU discharge. The differences in the predictive validity of the functional outcomes at the two time points may relate to differences in the individual constructs measured within each measure. The PFIT-s involves evaluation of peripheral muscle strength (shoulder flexion and knee extension); sit to stand assistance and marching in place cadence. In contrast the IMS and FSS-ICU examine a hierarchical level of dependence/assistance required to perform functional tasks such as bed mobility, sitting, standing and walking. Physical function is a complex entity to measure and can encompass a range of different constructs. Therefore, in the future it is important that within functional tests we consider what the individual constructs of a functional test are able to tell us about a patient's future trajectory of recovery and the prediction of discharge to home and resumption of family, societal and community roles.

In addition to consideration of the clinimetric properties of outcome measures it is important to consider other aspects of utility such as time to complete the measure, equipment and training required or health professional expertise, and availability of the outcome measure for use in clinical practice. The advantage of the four functional measures examined within this study is that they are readily available, and require little dedicated equipment (for example, for the PFIT-s a stopwatch is required for marching in place cadence, or gait aids/ chairs if required for ambulation/sitting out of bed components of all functional tests). All outcome measures take $<20$ minutes to complete.

Interventions aimed at improving functional recovery may not only minimize or improve physical function but may also affect cognitive processing, and emotional health. Therefore measures that evaluate these aspects also need to be examined across different time points in the trajectory of recovery $[2,41]$. It is important that there is mapping of outcome measures within the ICF framework to capture impairment, activity limitation and participation restrictions across the continuum of recovery. It is likely in the future that there will be overlap in the functional outcomes that are utilized, which enable sensitive monitoring of functional recovery and determination of the efficacy of interventional strategies.

The ICU environment is a challenging setting in which to conduct research, due to patient heterogeneity, severity of illness, and mortality. To improve the ability to compare findings between research studies, there is an urgent need to adopt a standardized core set of outcome measures. Functional recovery and independence is complex and requires individuals to master multiple facets simultaneously [2,41]. For example independent mobility in the community requires not only muscular strength, but postural control, endurance, cognitive processing to anticipate obstacles and respond to the changing demands of the environment surrounding them $[2,41]$. It is therefore important that outcome measures are adopted that are setting-specific to ensure improvement and/or deterioration in function are meaningfully encapsulated to capture changes in impairment, activity limitations and participation restrictions. For example, the distance a patient ambulates and level of assistance does not provide you with information on the quality of the patient's ambulation. We hypothesise that there will not be a single functional outcome that can be utilized across the continuum of recovery post critical illness. It is also important to consider different stages of recovery, as this will vary from patient to patient at different time points. For example, some patients are able to complete a $6 \mathrm{MWT}=$ six minute walk test at hospital discharge, while others cannot. This will enable the identification of deficits, which may impact on the ability to discharge home and ultimately resume family and societal roles in individuals with very low levels of function, through to higher-functioning individuals after the insult of initial critical illness.

\section{Limitations}

While combining data from these two studies improved the generalizability of findings, the overall sample size 
was small. This study was underpowered to examine the clinimetric properties of the SPPB and thus, results should be viewed with caution and interpreted as an overall trend in findings. Whilst reliability of the functional outcomes was not examined specifically within this study, the reliability of the PFIT-s and IMS has been previously reported within individuals with critical illness $[17,42]$. Currently there are no published data on the reliability of the FSS-ICU and this is an area that needs to be addressed in future research. To our knowledge, the reliability of the SPPB scoring has not been reported for individuals with critical illness; it has been shown to have excellent reliability in the general geriatric population [43]. Results from logistic regression need to be validated in an independent sample, and therefore, results on the ability of the PFIT-s, FSS-ICU and IMS to predict discharge destination must be viewed with caution.

This study only examined functional measures within the ICU setting, and the utility of these outcomes in the post ICU setting warrants further examination. The CPAx, Perme mobility and SOMS scales were not examined within this paper and warrant further testing to determine their utility for measuring functional changes within individuals with critical illness. It is essential that rigorous examination of currently utilized functional measures continue to be undertaken in order to determine the most appropriate outcome/s, which can be utilized across the continuum of patient recovery specifically for individuals with critical illness.

\section{Conclusions}

There is excellent criterion validity for other functional measures (FSS-ICU, IMS and SPPB) against the PFIT-s in the ICU setting. Higher PFIT-s scores on awakening were predictive of discharge directly home. All tests were responsive to change, however, the SPPB and IMS were limited by floor effects when used in the ICU. Based on the findings in this study the PFIT-s and FSSICU are promising functional measures and should be considered currently when measuring physical function in the ICU in clinical practice and research.

\section{Key messages}

- Impairment in physical function is a significant problem for survivors of critical illness.

- PFIT-s and FSS-ICU are promising functional measures and should be considered when measuring physical function in the ICU.

- A core set of outcome measures, which map impairment, activity limitations and participation restrictions within the ICF framework need to be developed, which can be utilized across different time points of recovery.

\section{Additional file}

Additional file 1: Table S1. Logistic regression models for prediction of discharge directly home. D/C, discharge; FSS-ICU, Functional Status Score for the Intensive Care Unit; IMS, ICU mobility scale; n, number; PFIT-s, Physical Function in Intensive Care Test scored.

\section{Abbreviations}

APACHE II: Acute physiological and chronic health evaluation 2; COSMIN: COnsensus-based standards for the selection of health status Measurement INstruments; CPAx: Chelsea critical care physical assessment tool; FSS-ICU: Functional status score for the intensive care unit; ICF: International classification of functioning; ICU-AW: intensive care unit-acquired weakness; IMS: ICU mobility scale; LOS: length of stay; MCID: minimal clinically important difference; MID: minimal important difference; MRC: Medical Research Council; MV: Mechanical ventilation; PFIT-s: Physical function in intensive care test scored; SEM: standard error of measurement; SPPB: Short physical performance battery; STROBE: Strengthening the reporting of observational studies in epidemiology.

\section{Competing interests}

The authors declare that they have no competing interests.

\section{Authors' contributions}

SP contributed to the design of the study and was responsible for data analysis and interpretation and drafted, revised and agreed on the final manuscript version of the submission. Authors CG and LD contributed to the design of the study, data analysis and interpretation of the results and contributed to the manuscript revision. Authors SB, LB and HW contributed to data acquisition and manuscript revision. All authors read and approved the final manuscript.

\section{Acknowledgements}

The authors would like to acknowledge the support of the Physiotherapy Departments at Austin Health and Royal Melbourne Hospital in the undertaking of this study.

\section{Author details}

${ }^{1}$ Department of Physiotherapy, The University of Melbourne, Level 7 Alan Gilbert Building, 161 Barry St, Parkville, 3010 Melbourne, VIC, Australia. ${ }^{2}$ Department of Physiotherapy, Melbourne Health, 3050 Melbourne, VIC, Australia. ${ }^{3}$ Institute for Breathing and Sleep, 3084 Melbourne, VIC, Australia.

${ }^{4}$ Department of Physiotherapy, Austin Health, 3084 Melbourne, VIC, Australia.

Received: 18 December 2014 Accepted: 20 February 2015

Published online: 29 March 2015

\section{References}

1. Herridge M, Tansey CM, Matté A, Tomlinson G, Diaz-Granados N, Guest CB, et al. Functional disability 5 years after acute respiratory distress syndrome. N Engl J Med. 2011;364:1293-304.

2. Hough C. Improving function during and after critical care. Curr Opin Crit Care. 2013;19:488-95.

3. Iwashyna T. Survivorship will be the defining challenge of critical care in the 21st century - Editorial. Ann Intern Med. 2010;153:204-5.

4. Bruce B, Fries JF, Lingala B, Gandek B, Rose M, Ware Jr JE. Better assessment of physical function: item improvement is neglected but essential. Arthritis Res Ther. 2009;11:R191.

5. Iwashyna T. Trajectories of recovery and dysfunction after acute illness, with implications for clinical trial design. Am J Respir Crit Care Med. 2012;186:302-4.

6. Parry SM, Granger CL, Berney S, Jones J, Beach L, El-Ansary D, et al. Assessment of impairment and activity limitations in the critically ill: A systematic review of measurement instruments and their clinimetric properties. Intensive Care Med. 2015. [Epub ahead of print].

7. de Vet $\mathrm{H}$, Terwee CB, Mokkink LB, Knol DL. Measurement in Medicine - A Practical Guide. Cambridge: Cambridge University Press; 2011.

8. Portney L. Foundations of clinical research: applications to practice. Vol. 2nd ed. Watkins MP, editor. Pearson/Prentice Hall: Upper Saddle River, NJ; 2000. 
9. de Vet HC, Terwee CB, Ostelo RW, Beckerman H, Knol DL, Bouter LM. Minimal changes in health status questionnaires: distinction between minimally detectable change and minimally important change. Health Qual Life Outcomes. 2006:4:54

10. Granger C, McDonald CF, Parry SM, Oliveira CC, Denehy L. Functional capacity, physical activity and muscle strength assessment of individuals with non-small cell lung cancer: a systematic review of instruments and their measurement properties. BMC Cancer. 2013;13:135.

11. Elliott D, Denehy L, Berney S, Alison JA. Assessing physical function and activity for survivors of a critical illness: a review of instruments. Aust Crit Care. 2011;24:155-66.

12. Connolly BA, Jones GD, Curtis AA, Murphy PB, Douiri A, Hopkinson NS, et al, Clinical predictive value of manual muscle strength testing during critical illness: an observational cohort study. Crit Care. 2013;17:R229.

13. Denehy L, de Morton NA, Skinner EH, Edbrooke L, Haines K, Warrillow S, et al. A physical function test for use in the intensive care unit: validity, responsiveness, and predictive utility of the physical function ICU test (scored). Phys Ther. 2013;9312:1638-45.

14. Corner EJ, Wood H, Englebretsen C, Thomas A, Grant RL, Nikoletou D, et al The Chelsea critical care physical assessment tool (CPAx): validation of an innovative new tool to measure physical morbidity in the general adult critical care population; an observational proof-of-concept pilot study. Physiotherapy. 2013;99:33-41.

15. Nawa R, Lettvin C, Winkelman C, Evora PR, Perme C, et al. Initial inter-rater reliability for a novel measure of patient mobility in a Cardiovascular ICU. J Crit Care. 2014;29:475.e1-5.

16. Kasotakis G, Schmidt U, Perry D, Grosse-Sundrup M, Benjamin J, Ryan C, et al. The surgical intensive care unit optimal mobility score predicts mortality and length of stay. Crit Care Med. 2012;40:1122-8.

17. Hodgson C, Needham D, Haines K, Bailey M, Ward A, Harrold M, et al. Feasibility and inter-rater reliability of the ICU Mobility Scale. Heart Lung. 2014;43:19-24.

18. Zanni JM, Korupolu R, Fan E, Pradhan P, Janjua K, Palmer JB, et al. Rehabilitation therapy and outcomes in acute respiratory failure: an observational pilot project. J Crit Care. 2010;25:254-62.

19. Thrush A, Rozek M, Dekerlegand J. The clinical utility of the functional status score for the intensive care unit (FSS-ICU) at a long-term acute care hospital: a prospective cohort study. Phys Ther. 2012;92:1536-45.

20. Files D, Morris P, Shrestha S, Dhar S, Young M, Hauser J, et al. Randomised, controlled pilot study of early rehabilitation strategies in acute respiratory failure. Crit Care. 2013;17:P540.

21. Needham DM, Dinglas VD, Morris PE, Jackson JC, Hough CL, Mendez-Tellez PA, et al. Physical and cognitive performance of patients with acute lung injury 1 year after initial trophic versus full enteral feeding. EDEN trial follow-up. Am J Respir Crit Care Med. 2013;188:567-76.

22. Guralnik JM, Simonsick EM, Ferrucci L, Glynn RJ, Berkman LF, Blazer DG, et al. A short physical performance battery assessing lower extremity function: association with self-reported disability and prediction of mortality and nursing home admission. J Gerontol. 1994;49:M85-94.

23. Perera S, Mody SH, Woodman RC, Studenski SA. Meaningful change and responsiveness in common physical performance measures in older adults. J Am Geriatr Soc. 2006;54:743-9.

24. Nordon-Craft A, Schenkman M, Edbrooke L, Malone DJ, Moss M, Denehy L. The physical function intensive care test: implementation in survivors of critical illness. Phys Ther. 2014;94:1499-507.

25. Corner EJ, Soni N, Handy JM, Brett SJ. Construct validity of the Chelsea critical care physical assessment tool: an observational study of recovery from critical illness. Crit Care. 2014;18:R55.

26. Berney S, Haines K, Skinner EH, Denehy L. Safety and feasibility of an exercise prescription approach to rehabilitation across the continuum of care for survivors of critical illness. Phys Ther. 2012;92:1524-35.

27. Mokkink LB, Terwee CB, Knol DL, Stratford PW, Alonso J, Patrick DL, et al. The COSMIN checklist for evaluating the methodological quality of studies on measurement properties: a clarification of its content. BMC Med Res Methodol. 2010;10:22.

28. von Elm E, Altman DG, Egger M, Pocock SJ, Gøtzche PC, Vandenbroucke JP, et al. The Strengthening the Reporting of Observational Studies in Epidemiology (STROBE) Statement: guidelines for reporting observational studies. Bull World Health Organ. 2007:85:867-72.

29. De Jonghe B, Sharshar T, Lefaucheur JP, Authier F-J, Durand-Zaleski I, Boussarsar $M$, et al. Paresis acquired in the intensive care unit: a prospective multicenter study. J Am Med Assoc. 2002;288:2859-67.
30. Hough C, Lieu B, Caldwell E. Manual muscle strength testing of critically ill patients: feasibility and interobserver agreement. Crit Care. 2011;15:R43.

31. Nordon-Craft A, Schenkman M, Edbrooke L, Malone DJ, Moss M, Denehy L. The physical function intensive care test: implementation in survivors of critical illness. Phys Ther. 2014;94:1-9.

32. Terwee CB, Mokkink LB, van Poppel MN, Chinapaw MH, van Mechelen W, de Vet HC. Qualitative attributes and measurement properties of physical activity questionnaires: a checklist. Sports Med. 2010;40:525-37.

33. Altman DG. Practical statistics for medical research. London: CRC/Chapman and Hall; 1991.

34. Cohen J. Statistical power analysis for the behavioral sciences. 2nd ed. Hillsdale (NJ): Lawrence Erlbaum Associates; 1988.

35. Husted JA, Cook RJ, Farewell VT, Gladman DD. Methods for assessing responsiveness: a critical review and recommendations. J Clin Epidemiol. 2000;53:459-68

36. Norman G, Sloan J, Wyrwich K. Interpretation of changes in healthrelated quality of life: the remarkable universality of half a standard deviation. Med Care. 2003;41:582-92

37. Guyatt G, Walter $\mathrm{S}$, Norman G. Measuring change over time: assessing the usefulness of evaluative instruments. J Chronic Dis. 1987;40:171-8.

38. Terwee CB, Bot SD, de Boer MR, van der Windt DA, Knol DL, Dekker J, et al. Quality criteria were proposed for measurement properties of health status questionnaires. J Clin Epidemiol. 2007:60:34-42.

39. Guyatt GH, Osoba D, Wu AW, Wyrwich KW, Norman GR. Clinical Significance Consensus Meeting Group. Methods to explain the clinical significance of health status measures Mayo Clin Proc. 2002;77:371-83.

40. Johnson M, Bland JM, Oxberry SG, Abernethy AP, Currow DC. Clinically important differences in the intensity of chronic refractory breathlessness. J Pain Symptom Manage. 2013;46:957-63.

41. Herridge M, Batt J, Dos Santos C. ICU-acquired weakness, morbidity and death. Am J Respir Crit Care Med. 2014;190:361-2.

42. Skinner EH, Berney S, Warrillow S, Denehy L. Development of a physical function outcome measure (PFIT) and a pilot exercise training protocol for use in intensive care. Crit Care Resusc. 2009;11:110-5.

43. Ostir GV, Volpato S, Fried LP, Chaves P, Guralnik JM. Women's Health and Aging Study. Reliability and sensitivity to change assessed for a summary measure of lower body function: results from the Women's Health and Aging Study. J Clin Epidemiol. 2002;55:916-21.

\section{Submit your next manuscript to BioMed Central and take full advantage of:}

- Convenient online submission

- Thorough peer review

- No space constraints or color figure charges

- Immediate publication on acceptance

- Inclusion in PubMed, CAS, Scopus and Google Scholar

- Research which is freely available for redistribution 